\title{
Classical versus Quantum Time Evolution of Densities at Limited Phase-Space Resolution
}

\author{
Christopher Manderfeld ${ }^{1}$, Joachim Weber ${ }^{1,2}$, and Fritz Haake ${ }^{1}$ \\ 1 Fachbereich Physik, Universität Essen, 45117 Essen, Germany \\ 2 Department of Physics of Complex Systems, Weizmann Institute of Science, Rehovot 76100, Israel
}

\begin{abstract}
We study the interrelations between the classical (Frobenius-Perron) and the quantum (Husimi) propagator for phase-space (quasi-)probability densities in a Hamiltonian system displaying a mix of regular and chaotic behavior. We focus on common resonances of these operators which we determine by blurring phase-space resolution [1, A]. We demonstrate that classical and quantum time evolution look alike if observed with a resolution much coarser than a Planck cell and explain how this similarity arises for the propagators as well as their spectra. The indistinguishability of blurred quantum and classical evolution implies that classical resonances can conveniently be determined from quantum mechanics and in turn become effective for decay rates of quantum correlations.
\end{abstract}

\section{INTRODUCTION}

Classical Hamiltonian motion can appear irreversible, as is most familiar for many-body systems but also observed for chaos of two degrees of freedom. Effective irreversibility can show up as relaxation of certain correlation functions, and the decay rates involved are resonances of the propagator for phase-space densities known as the Frobenius-Perron operator. Frobenius-Perron resonances have recently been shown to play a key role for the interrelations of classical, semiclassical and quantum dynamics, e.g. in a superanalytic approach to universal fluctuations in quantum (quasi-) energy spectra 3 [5].The purpose of this paper is to substantiate those interrelations.

A quantum analog to the Frobenius-Perron operator is the propagator for Husimi functions. Husimi functions are positive and normalized distributions in phase space and turn into phase-space densities in the classical limit. Both classical densities and Husimi functions can be approximated within the same Hilbert space of phase-space functions. As a caveat we remark here that in the quantum case this Hilbert space of phase-space functions is not to be confused with the Hilbert space of quantum wave functions.

Even though without coupling to external reservoirs both the classical and the quantum dynamics are reversible, both do appear effectively irreversible when phase-space structures cannot be fully resolved. Since any measurement of phase-space variables can be conducted with finite precision only, restricted phase-space resolution is rather ubiquitous in the macroworld; in particular for classical chaotic dynamics, where phase-space structures extend over an infinite hierarchy of scales, but also in the quantum case, if a Planck cell is too small to be resolved.

By adopting finite phase-space resolution much coarser than a Planck cell for a prototypical dynamics we show that the quantum propagator becomes indistinguishable from the classical propagator. In particular, we find that resonances of the two propagators coincide, indicating that the same "relaxation processes" occur in classical and quantum dynamics. These processes and thus the resonances can be linked to the resolved structures in phase space 1.2.2. In particular, in as much as resonances are related to localized structures in phase space, our results suggest that resonances may cause some quantum eigenfunctions to assign exceptional weight to certain regions in phase or configuration space and possibly be one of the origins of scarring. This line of thought will be taken up in a separate paper.

If the classical phase space displays a mix of regular and chaotic structures, along with effective irreversible behavior caused by hyperbolicity, we also encounter recurrences due to elliptic islands in phase space; the latter manifest themselves as (almost) unimodular eigenvalues of the Frobenius-Perron and Husimi propagators.

\section{CLASSICAL DYNAMICS ON THE SPHERE}

As a prototypical Hamiltonian system with a mixed phase space we consider a periodically kicked angular momentum vector

$$
\mathbf{J}=(j \sin \theta \cos \varphi, j \sin \theta \sin \varphi, j \cos \theta)
$$

of conserved length $j$, also known as the kicked top. Such a system has one degree of freedom, and its phase space is the sphere, with the "azimuthal" angle $\varphi$ as the coordinate and the cosine of the "polar" angle $\theta$ as the conjugate 
momentum. The dynamics is specified as a stroboscopic area-preserving map $M$ on phase space. We choose the dynamics to consist of rotations $R_{z}\left(\beta_{z}\right), R_{y}\left(\beta_{y}\right)$ about the $y-$ and $z$-axes by angles $\beta_{y}$, $\beta_{z}$ and a "torsion", i.e. a nonlinear rotation $T_{z}(\tau)=R_{z}(\tau \cos \theta)$ about the $z$-axis which changes $\varphi$ by $\tau \cos \theta$,

$$
M=T_{z}(\tau) R_{z}\left(\beta_{z}\right) R_{y}\left(\beta_{y}\right) .
$$

With $\beta_{z}$ and $\beta_{y}$ fixed, we can control the degree of chaoticity of the dynamics by varying $\tau$. While the dynamics is integrable for $\tau=0$, with increasing $\tau$ chaoticity sets in, until for $\tau=10$ elliptic islands have become so small that they are difficult to detect.

In the Liouville picture the time evolution of a phase-space density $\rho$ is governed by Liouville's equation,

$$
\partial_{t} \rho=\mathcal{L} \rho=\{H, \rho\}
$$

where the Liouville operator $\mathcal{L}$, the Poisson bracket with the Hamiltonian $H$, appears as the generator. For our rotations and torsion on the sphere we must separately take $H=\beta_{y} J_{y}, H=\beta_{z} J_{z}$, and $H=\frac{\tau}{2} J_{z}^{2}$. Denoting the corresponding Liouvillians by $\mathcal{L}_{R_{y}}, \mathcal{L}_{R_{z}}, \mathcal{L}_{T_{z}}$ we imagine Liouville's equation for each of them separately integrated over a unit time span. The product of the resulting three propagators yields the Frobenius-Perron operator $\mathcal{P}=$ $\exp \left(\mathcal{L}_{T_{z}}\right) \exp \left(\mathcal{L}_{R_{z}}\right) \exp \left(\mathcal{L}_{R_{y}}\right)$

While a phase-space density is usually considered as $L^{1}$-integrable, it can be assumed to belong to a Hilbert space of $L^{2}$-functions as well. In this Hilbert space $\mathcal{P}$ is represented by an infinite dimensional unitary matrix. Accordingly its spectrum is unimodular and, depending on the character of the dynamics, may consist of both discrete eigenvalues and continuous parts. The complete basis to represent the matrix in may be chosen as ordered with respect to phase-space resolution, to eventually allow for a truncation of $\mathcal{P}$ to finite size in a systematic manner.

On the unit sphere the spherical harmonics $Y_{l m}(\theta, \varphi)$ with $l=0,1,2, \ldots$ and $|m| \leq l$ form a suitable basis. Employing that represenation resonances were identified in Ref. 11.2]. We here briefly review those results that are of relevance for our present discussion.

Phase-space resolution is characterized by the index $l$ : By using all $Y_{l m}$ with $0 \leq l \leq l_{\text {max }}$ phase-space structure of area $\propto 1 / l_{\max }^{2}$ can be resolved. Our classical Liouvillians can be written in terms of the differential operators $\hat{L}_{y}, \hat{L}_{z}$ well known from quantum mechanical contexts in the $Y_{l m}$ representation,

$$
\begin{aligned}
& \mathcal{L}_{R_{z}}=-\mathrm{i} \beta_{z} \hat{L}_{z}, \quad \hat{L}_{z}=-\mathrm{i} \frac{\partial}{\partial \varphi} \\
& \mathcal{L}_{R_{y}}=-\mathrm{i} \beta_{y} \hat{L}_{y}, \quad \hat{L}_{y}=-\mathrm{i}\left(-\cos \varphi \frac{\partial}{\partial \theta}+\cot \theta \cos \varphi \frac{\partial}{\partial \varphi}\right), \\
& \mathcal{L}_{T_{z}}=-\mathrm{i} \tau \cos \theta \hat{L}_{z} .
\end{aligned}
$$

The Frobenius-Perron operator of the $z$-rotation becomes the matrix

$$
\begin{aligned}
\left(\exp \left(\mathcal{L}_{R_{z}}\right)\right)_{l m, l^{\prime} m^{\prime}} & =\int \mathrm{d} \theta \sin \theta \mathrm{d} \varphi Y_{l m}^{*}(\theta, \varphi) \exp \left(-\beta_{z} \frac{\partial}{\partial \varphi}\right) Y_{l^{\prime} m^{\prime}}(\theta, \varphi) \\
& =\delta_{l l^{\prime}} \delta_{m m^{\prime}} \exp \left(-\mathrm{i} m \beta_{z}\right)
\end{aligned}
$$

which is diagonal in both indices. The Frobenius-Perron matrix of the $y$-rotation is blockdiagonal (diagonal in $l$ but not in $m$ ) and consists of the Wigner d-matrices well known from quantum mechanics as

$$
\left(\exp \left(\mathcal{L}_{R_{y}}\right)\right)_{l m, l^{\prime} m^{\prime}}=\delta_{l l^{\prime}} \mathrm{d}_{m m^{\prime}}^{l}\left(\beta_{y}\right)
$$

Finally, for the $z$-torsion the Frobenius-Perron matrix elements are finite sums over products of spherical Bessel functions $j_{l}(x)$ and Clebsch-Gordan coefficients,

$$
\left(\exp \left(\mathcal{L}_{T_{z}}\right)\right)_{l m, l^{\prime} m^{\prime}}=\delta_{m m^{\prime}}(-1)^{m} \sqrt{(2 l+1)\left(2 l^{\prime}+1\right)} \sum_{l^{\prime \prime}=\left|l-l^{\prime}\right|}^{l+l^{\prime}}(-\mathrm{i})^{l^{\prime \prime}} j_{l^{\prime \prime}}(m \tau) C_{000}^{l l^{\prime} l^{\prime \prime}} C_{-m m^{\prime} 0}^{l l^{\prime} l^{\prime \prime}} .
$$

While both rotation matrices are diagonal in the indices $l, l^{\prime}$, the torsion matrix $\mathcal{T}_{z}$ is diagonal in $m, m^{\prime}$. Therefore the elements of the Frobenius-Perron matrix for the composite dynamics are the products

$$
\mathcal{P}_{l m, l^{\prime} m^{\prime}}=\left(\exp \left(\mathcal{L}_{T_{z}}\right)\right)_{l m, l^{\prime} m}\left(\exp \left(\mathcal{L}_{R_{z}}\right)\right)_{l^{\prime} m, l^{\prime} m}\left(\exp \left(\mathcal{L}_{R_{y}}\right)\right)_{l^{\prime} m, l^{\prime} m^{\prime}},
$$

and no infinite sum hinders their evaluation. 


\section{QUANTUM EVOLUTION OF HUSIMI FUNCTIONS}

In the quantum mechanical description of the kicked top a wave vector $\left|\psi_{n}\right\rangle$ is propagated by a Floquet operator $F$ as $\left|\psi_{n+1}\right\rangle=F\left|\psi_{n}\right\rangle$ over one period in between two kicks. Again $F=T_{z}(\tau) R_{z}\left(\beta_{z}\right) R_{y}\left(\beta_{y}\right)$ consists of rotation and torsion operators that are built of components of an angular momentum vector $\hat{\mathbf{J}}=\left(\hat{J}_{x}, \hat{J}_{y}, \hat{J}_{z}\right)[\hat{b}]$ as

$$
\begin{aligned}
\hat{T}_{z}(\tau) & =\exp \left(-\mathrm{i} \frac{\tau}{2 j+1} \hat{J}_{z}^{2}\right), \\
\hat{R}_{z}\left(\beta_{z}\right) & =\exp \left(-\mathrm{i} \beta_{z} \hat{J}_{z}\right) \\
\hat{R}_{y}\left(\beta_{y}\right) & =\exp \left(-\mathrm{i} \beta_{y} \hat{J}_{y}\right) .
\end{aligned}
$$

An obvious choice for the basis of the Hilbert space of wave functions are the $(2 j+1)$ eigenvectors of $\hat{J}_{z}, \hat{J}_{z}|j m\rangle=$ $m|j m\rangle$ with fixed $j$ and $-j \leq m \leq j$. While $\hat{T}_{z}(\tau)$ and $\hat{R}_{z}\left(\beta_{z}\right)$ are diagonal matrices in this representation, $\hat{R}_{y}$ is again given by the Wigner d-matrix $\mathrm{d}^{j}\left(\beta_{y}\right)$. Once again we would like to emphasize that the Hilbert space of quantum wave functions must not be confused with the Hilbert space of functions on the classical phase space employed in Sec.II. (The distinction between the two Hilbert spaces becomes especially important when one associates, for integer values of $j$, the vectors $|j m\rangle$ with the spherical harmonics $Y_{j m}$ and the components of $\hat{\mathbf{J}}$ with differential operators.)

As the quantum mechanical analogue to classical phase-space densities and the Frobenius-Perron operator we consider Husimi functions and their propagator. A Husimi function turns into a classical density in the classical limit. The Husimi function $Q_{\rho}$ of a density operator $\rho$ is obtained as its diagonal matrix element with respect to a coherent state [7. 9]. Coherent states $|j \theta \varphi\rangle$ on the sphere assign to the observable $\hat{\mathbf{J}}$ a direction characterized by the angles $\theta$ and $\varphi$, but contrary to the classical description that direction is only specified up to the minimum uncertainty permitted by the angular momentum commutation relations. The relative variance $\left(\left\langle\hat{\mathbf{J}}^{2}\right\rangle-\langle\hat{\mathbf{J}}\rangle^{2}\right) / j^{2}=1 / j$ vanishes as the effective Planck constant $1 / j$ goes to zero in the classical limit. Any coherent state $|j \theta \varphi\rangle$ can be obtained via a rotation $\hat{R}(\theta, \varphi)$ from the coherent state $|j, m=j\rangle$ as $|j \theta \varphi\rangle=\hat{R}(\theta, \varphi)|j j\rangle$. The Husimi function thus reads

$$
Q_{\rho}(\theta, \varphi)=\langle j \theta \varphi|\rho| j \theta \varphi\rangle
$$

it can be expanded in terms of spherical harmonics,

$$
Q_{\rho}=\sum_{l=0}^{2 j} \sum_{m=-l}^{l} q_{l m} Y_{l m},
$$

with the index $l$ limited to $0 \leq l \leq 2 j$ [10]. The finiteness of the latter expansion will be commented on presently.

As a consequence, contrary to classical densities, $Q_{\rho}$ can be represented as a finite-dimensional vector with the dimension set by the effective (inverse) Planck constant $j$ as $(2 j+1)^{2}$. Since no structures smaller than a Planck cell can be resolved, the Husimi propagator should be expected to be a finite matrix.

The time evolution of $Q_{\rho}$ is governed by von Neumann's equation

$$
\partial_{t} Q_{\rho}(\theta, \varphi)=\mathcal{G} Q_{\rho}(\theta, \varphi)=-\mathrm{i}\langle j \theta \varphi|[\hat{H}, \rho]| j \theta \varphi\rangle
$$

with $\hat{H}=\frac{\tau}{2 j+1} \hat{J}_{z}^{2}, \hat{H}=\beta_{z} \hat{J}_{z}$ and $\hat{H}=\beta_{y} \hat{J}_{y}$ for torsion and rotations, respectively. In the representation (11) the generators $\mathcal{G}$ can be written as differential operators very similar in structure to the classical Liouvillians (任) as (see Appendix A)

$$
\begin{aligned}
& \mathcal{G}_{T_{z}}=-\mathrm{i} \tau\left(\cos \theta-\frac{1}{2 j+1} \frac{\partial}{\partial \theta} \sin \theta\right) \hat{L}_{z}, \\
& \mathcal{G}_{R_{z}}=-\mathrm{i} \beta_{z} \hat{L}_{z}, \\
& \mathcal{G}_{R_{y}}=-\mathrm{i} \beta_{y} \hat{L}_{y} .
\end{aligned}
$$

It is seen that the quantum Husimi generators $\mathcal{G}$ and the classical Liouvillians $\mathcal{L}$ are identical for rotations, while for the $z$-torsion a quantum correction arises in $\mathcal{G}_{T_{z}}$, which is formally of order $(2 j+1)^{-1}$. This correction is responsible for all the difference between classical and quantum mechanics in the sequel. We should point out here that the 
correction in question ensures the finite range of the Husimi matrix mentioned before, $0 \leq l \leq 2 j,-l \leq m \leq l$. In contrast to the rotations, the torsion couples different angular momenta $l$. The action of the torsion generator on the spherical harmonics is calculated in Appendix B as

$$
\begin{aligned}
\mathcal{G}_{T_{z}} Y_{l m}= & -\mathrm{i} \frac{m \tau}{2 j+1}\left((2 j-l) \sqrt{\frac{(l+m+1)(l-m+1)}{(2 l+1)(2 l+3)}} Y_{l+1, m}\right. \\
& \left.+(2 j+1+l) \sqrt{\frac{(l+m)(l-m)}{(2 l+1)(2 l-1)}} Y_{l-1, m}\right) .
\end{aligned}
$$

The generator couples spherical harmonics of neighbouring $l$, except for the cases $l=0$ and $l=2 j$, where the prefactors of $Y_{l-1, m}$ and $Y_{l+1, m}$ vanish, respectively.

It follows from the foregoing remarks on the Husimi generator that the Husimi propagators $\exp \left(\mathcal{G}_{R_{z}}\right)$, $\exp \left(\mathcal{G}_{R_{y}}\right)$ for rotations are identical to their Frobenius-Perron correspondents. However, the torsion propagator $\exp \left(\mathcal{G}_{T_{z}}\right)$ still has to be determined. Clearly, the $(2 j+1)^{2}$ "Husimi functions" $Q_{\left|j m_{1}\right\rangle\left\langle j m_{2}\right|}=\left\langle j \theta \varphi \mid j m_{1}\right\rangle\left\langle j m_{2} \mid j \theta \varphi\right\rangle$ are eigenfunctions of $\exp \left(\mathcal{G}_{T_{z}}\right)$ with eigenvalues $\exp \left(-\mathrm{i} \tau\left(m_{1}^{2}-m_{2}^{2}\right) /(2 j+1)\right)$. Note that the $Q_{\left|j m_{1}\right\rangle\left\langle j m_{2}\right|}$ represents the skew ket-bras $\left|j m_{1}\right\rangle\left\langle j m_{2}\right|$ in the sense of the definition (10). In order to proceed to representing $\exp \left(\mathcal{G}_{T_{z}}\right)$ in the basis $\left\{Y_{l m}\right\}$ we have constructed the linear transformation relating the spherical harmonics $Y_{l m}$ to the "Husimi functions" $Q_{\left|j m_{1}\right\rangle\left\langle j m_{2}\right|}$ (see Appendix C). Like its classical counterpart $\exp \left(\mathcal{L}_{T_{z}}\right)$ (see (7)) the Husimi propagator for $z$-torsion, $\exp \left(\mathcal{G}_{T_{z}}\right)$, turns out diagonal in $m$ but not in $l$. As a consequence, the Husimi matrix elements are simple products of torsion and rotation matrix elements,

$$
\mathcal{U}_{l m, l^{\prime} m^{\prime}}=\left(\exp \left(\mathcal{G}_{T_{z}}\right)\right)_{l m, l^{\prime} m}\left(\exp \left(\mathcal{G}_{R_{z}}\right)\right)_{l^{\prime} m, l^{\prime} m}\left(\exp \left(\mathcal{G}_{R_{y}}\right)\right)_{l^{\prime} m, l^{\prime} m^{\prime}} .
$$

\section{CLASSICAL TIME EVOLUTION AT FINITE PHASE-SPACE RESOLUTION}

As has been illustrated for the kicked top in two previous papers [1,2], resonances and eigenvalues of the FrobeniusPerron operator as well as the associated phase-space structures can be found when time evolution is looked upon with finite resolution. However, since our aim is to compare classical and quantum dynamics at limited phasespace resolution, we briefly summarize the results. In representations with respect to the resolution-ordered basis functions $Y_{l m}$ with $l=0,1, \ldots, \infty$ and integer $|m| \leq l$, a limitation of phase-space resolution is achieved by a simple truncation, discarding all matrix elements of $\mathcal{P}_{l m, l^{\prime} m^{\prime}}$ with $l, l^{\prime}>l_{\max }$. The resulting $N \times N$ matrix $\mathcal{P}^{(N)}$ of dimension $N=\left(l_{\max }+1\right)^{2}$ is a nonunitary approximation to the unitary $\mathcal{P}$ with a purely discrete spectrum bounded in modulus by unity. An investigation of the $N$-dependence of the eigenvalues of $\mathcal{P}^{(N)}$ reveals that with increasing resolution some eigenvalues persist in their positions, either close to or well inside the unit circle. Of these "frozen" eigenvalues the almost unimodular ones turn into unimodular eigenvalues of the unitary $\mathcal{P}$ as $N \rightarrow \infty$; the subunimodular eigenvalues reflect resonances of $\mathcal{P}$ in a higher Riemannian sheet. The different nature of "frozen" unimodular and subunimodular eigenvalues becomes more evident through an investigation of their eigenfunctions. For unimodular eigenvalues the eigenfunctions are sharply localized on islands of regular motion surrounding elliptic periodic orbits, while for subunimodular eigenvalues eigenfunctions are localized around unstable manifolds of hyperbolic periodic orbits. The condition for freezing of an eigenvalue evidently is that at least the largest associated phase-space structures are resolved. For the kicked top with $\tau=10, \beta_{z}=1$, and $\beta_{y}=1$ we propose to illustrate the freezing of eigenvalues. As no elliptic structures can be resolved even at the highest employed resolution, $l_{\max }=70$, all frozen eigenvalues except for the one at unity (pertaining to the stationary constant eigenfunction $Y_{00}$ ) lie well inside the unit circle. A gray-scale histogram of all eigenvalues with moduli greater than $1 / 4$ of all matrices $\mathcal{P}^{(N)}$ with $l_{\max }=20,21 \ldots, 70$ in the complex plane is shown in figure 1. Eigenvalues of smaller modulus have been rejected, since they have not settled yet and would spoil the histogram due to their large density near the origin. Dark areas in the histogram indicate large amplitudes and thus the positions of "frozen" eigenvalues. Several nonunimodular "frozen" eigenvalues are clearly visible. Their precise positions at resolutions $l_{\max }=30,40,50,60$, and 68 are given in table 


\section{QUANTUM TIME EVOLUTION AT BLURRED PHASE-SPACE RESOLUTION}

The smallest scale in phase space resolvable in Husimi functions is set by Planck's constant. Recalling that for the kicked top the role of the inverse Planck constant is played by $j$, in the Hilbert space spanned by the $Y_{l m}$ the Husimi propagator can be represented as a finite $N \times N$ matrix of dimension $N=(2 j+1)^{2}$. This is because with respect to the basis functions $Y_{l m}$ all matrix elements of $\mathcal{U}$ involving indices $l>2 j$ vanish. The $(2 j+1)^{2}$ discrete eigenvalues of $\mathcal{U}$ are of unit modulus due to the unitarity of Schrödinger's time evolution which also implies conservation of probability in phase space. With the eigenvalue problem for the Floquet operator $F\left|\phi_{k}\right\rangle=\exp \left(-\mathrm{i} \phi_{k}\right)\left|\phi_{k}\right\rangle$ solved, the eigenphases of the full $\mathcal{U}$ are the quasienergy differences $\left(\phi_{k}-\phi_{l}\right)$ and the associated eigenfunctions follow as $\left\langle j \theta \varphi \mid \phi_{k}\right\rangle\left\langle\phi_{l} \mid j \theta \varphi\right\rangle$.

We truncate the propagator $\mathcal{U}$ in the same manner as the Frobenius-Perron matrix, with the resolution parameter $l_{\max }$ taking a value $l_{\max }<2 j$. Now the dependence of the eigenvalues on the truncation parameter $l_{\max }$ can be studied. We are particularly interested in semiclassical propagators, i.e. large values of $j$. Introducing the ratio $\kappa=l_{\max } /(2 j), \kappa=1$ represents complete resolution and $\kappa \ll 1$ the limit with Planck cells far from resolved.

Starting from $\kappa=1$ and slowly decreasing $\kappa$, the eigenvalues of $\mathcal{U}$ feel the truncation as a small perturbation at first that slightly shifts them inside the unit circle. With a further decrease of $\kappa$, eigenvalues are spread over the entire unit disk. When $\kappa$ finally becomes small, the eigenvalues of the truncated propagator matrix coincide with the eigenvalues of the corresponding Frobenius-Perron matrix $\mathcal{P}^{(N)}$ of the same dimension. This is illustrated in figure 2, where the Husimi eigenvalues of the kicked top with $\tau=10, \beta_{z}=\beta_{y}=1$ for $\kappa=1,0.5,0.16$ and 0.08 are compared to the eigenvalues of the Frobenius-Perron matrix at $l_{\max }=32$. For the sake of the comparison we kept $l_{\max }=32$ fixed also for the quantum propagator and changed $\kappa$ by choosing different quantum numbers $j=16,32,100,200$. As the most striking aspect of the spectral coincidence at small $\kappa$, resonances as well as eigenvalues of the full Frobenius-Perron operator can be obtained from quantum dynamics by looking for frozen eigenvalues of the truncated Husimi propagator at different values $l_{\max }$ in the limit $\kappa \ll 1$. Furthermore, the coincidence of eigenvalues implies that also the associated eigenfunctions are in agreement. In particular, the eigenfunctions of the truncated Husimi propagator display the same strong scarring on unstable manifolds and elliptic islands in phase space as their classical counterparts.

The most drastic difference between the two propagator matrices lies, of course, in the finiteness of the quantum propagator. Moreover, the matrix elements with $l$ near the quantum cutoff $2 j$ differ appreciably: It is illustrated in Appendix B that the quantum correction of the torsion generator in the basis of spherical harmonics is of the order $l /(2 j+1)$ relative to the classical part. On the other hand, on large phase-space scales, i.e. for $l \ll 2 j$, the Frobenius-Perron and Husimi propagators become indistinguishable as $\kappa \rightarrow 0$. An expansion of the Husimi propagator in powers of $(2 j+1)^{-1}$ yields

$$
(\exp (\mathcal{G}))_{l m, l^{\prime} m^{\prime}}=(\exp (\mathcal{L}))_{l m, l^{\prime} m^{\prime}}+\frac{1}{2 j+1}\left(\sum \ldots\right)_{l m, l^{\prime} m^{\prime}} .
$$

Assuming $l_{\max }$ fixed, for $j \rightarrow \infty$ the quantum correction of a matrix element decays as $1 /(2 j+1)$. Accordingly, in this case we expect the mean squared deviation between the matrix elements of the two propagators to asymptotically vanish as $1 /(2 j+1)^{2}$. Figure 3 confirms that expectation.

\section{CONCLUSION}

In conclusion, for a prototypical dynamical system we have formulated time evolution in terms of phase-space distributions, both classically and quantum mechanically. The comparison of the two respective propagators at limited phase-space resolution unveils the classical character of the quantum dynamics on large phase-space scales. Only when quantum coherences on the scale of Planck's constant are resolved, the peculiarities of quantum mechanics arise. Due to this fact Frobenius-Perron resonances can be identified from the quantum propagator. Moreover, quantum propagation is described by a finite matrix and therefore more easily treated than the classical counterpart which is an infinite matrix. Our results on classical and quantum signatures of resonances might become helpful in explaining phase-space localization (scars) of quantum eigenfunctions.

We gratefully acknowledge support by the Sonderforschungsbereich 'Unordnung und große Fluktuationen' of the Deutsche Forschungsgemeinschaft and support of the Minerva foundation.

\section{APPENDIX}




\section{A. Husimi generators for rotation and torsion}

A normalized coherent state can be written as [7]

$$
|j \theta \varphi\rangle=\left(1+\alpha \alpha^{*}\right)^{-j} \mathrm{e}^{\alpha \hat{J}_{-}}|j j\rangle=\left(1+\alpha \alpha^{*}\right)^{-j} \sum_{m=-j}^{j} \alpha^{j-m} \sqrt{\left(\begin{array}{c}
2 j \\
j-m
\end{array}\right)}|j m\rangle
$$

where $\alpha$ is the complex parameter $\alpha=\tan \frac{\theta}{2} \mathrm{e}^{\mathrm{i} \varphi}$ and $\hat{J}_{ \pm}=\hat{J}_{x} \pm \mathrm{i} \hat{J}_{y}$ are the familiar ladder operators. In this manner a Husimi function (10) can be expressed as a function of $\alpha$ and its complex conjugate $\alpha^{*}$,

$$
Q_{\rho}\left(\alpha, \alpha^{*}\right)=\left(1+\alpha \alpha^{*}\right)^{-2 j}\left\langle j j\left|\mathrm{e}^{\alpha^{*} \hat{J}_{+}} \rho \mathrm{e}^{\alpha \hat{J}_{-}}\right| j j\right\rangle
$$

In order to derive the generators defined by von Neumann's equation (12) we start with the following two identities

$$
\begin{aligned}
\left\langle j j\left|\mathrm{e}^{\alpha^{*} \hat{J}_{+}} \rho \hat{J}_{z} \mathrm{e}^{\alpha \hat{J}_{-}}\right| j j\right\rangle & =\left\langle j j\left|\mathrm{e}^{\alpha^{*} \hat{J}_{+}} \rho \mathrm{e}^{\alpha \hat{J}_{-}}\left(\hat{J}_{z}-\alpha \hat{J}_{-}\right)\right| j j\right\rangle \\
& =\left(j-\alpha \frac{\partial}{\partial \alpha}\right)\left\langle j j\left|\mathrm{e}^{\alpha^{*} \hat{J}_{+}} \rho \mathrm{e}^{\alpha \hat{J}_{-}}\right| j j\right\rangle, \\
\left\langle j j\left|\mathrm{e}^{\alpha^{*} \hat{J}_{+}} \hat{J}_{z} \rho \mathrm{e}^{\alpha \hat{J}_{-}}\right| j j\right\rangle & =\left\langle j j\left|\left(\hat{J}_{z}-\alpha^{*} \hat{J}_{+}\right) \mathrm{e}^{\alpha^{*} \hat{J}_{+}} \rho \mathrm{e}^{\alpha \hat{J}_{-}}\right| j j\right\rangle \\
& =\left(j-\alpha^{*} \frac{\partial}{\partial \alpha^{*}}\right)\left\langle j j\left|\mathrm{e}^{\alpha^{*} \hat{J}_{+}} \rho \mathrm{e}^{\alpha \hat{J}_{-}}\right| j j\right\rangle .
\end{aligned}
$$

Thus, the generator for a rotation about the $z$-axis becomes

$$
\begin{aligned}
\frac{\mathrm{i}}{\beta_{z}} \mathcal{G}_{R_{z}} Q_{\rho} & =\left(1+\alpha \alpha^{*}\right)^{-2 j}\left\langle j j\left|\mathrm{e}^{\alpha^{*} \hat{J}_{+}}\left[\hat{J}_{z}, \rho\right] \mathrm{e}^{\alpha \hat{J}_{-}}\right| j j\right\rangle \\
& =\left(1+\alpha \alpha^{*}\right)^{-2 j}\left[\left(j-\alpha^{*} \frac{\partial}{\partial \alpha^{*}}\right)-\left(j-\alpha \frac{\partial}{\partial \alpha}\right)\right]\left\langle j j\left|\mathrm{e}^{\alpha^{*} \hat{J}_{+}} \rho \mathrm{e}^{\alpha \hat{J}_{-}}\right| j j\right\rangle .
\end{aligned}
$$

It is easy to see that the differential operator commutes with the prefactor $\left(1+\alpha \alpha^{*}\right)^{-2 j}$ such that

$$
\frac{\mathrm{i}}{\beta_{z}} \mathcal{G}_{R_{z}} Q_{\rho}=\left(\alpha \frac{\partial}{\partial \alpha}-\alpha^{*} \frac{\partial}{\partial \alpha^{*}}\right) Q_{\rho}
$$

Replacing the complex variables $\alpha, \alpha^{*}$ by the angular coordinates $\theta, \varphi$ we finally get

$$
\alpha \frac{\partial}{\partial \alpha}-\alpha^{*} \frac{\partial}{\partial \alpha^{*}}=-\mathrm{i} \frac{\partial}{\partial \varphi}
$$

whereupon the generator becomes

$$
\frac{\mathrm{i}}{\beta_{z}} \mathcal{G}_{R_{z}}=-\mathrm{i} \frac{\partial}{\partial \varphi}=\hat{L}_{z}
$$

Some more effort is required for the torsion generator,

$$
\mathrm{i} \frac{2 j+1}{\tau} \mathcal{G}_{T_{z}} Q_{\rho}=\left(1+\alpha \alpha^{*}\right)^{-2 j}\left\langle j j\left|\mathrm{e}^{\alpha^{*} \hat{J}_{+}}\left[\hat{J}_{z}^{2}, \rho\right] \mathrm{e}^{\alpha \hat{J}_{-}}\right| j j\right\rangle .
$$

After using (19) twice one obtains

$$
\begin{aligned}
\mathrm{i} \frac{2 j+1}{\tau} \mathcal{G}_{T_{z}} Q_{\rho} & =\left(1+\alpha \alpha^{*}\right)^{-2 j}\left[\left(j-\alpha^{*} \frac{\partial}{\partial \alpha^{*}}\right)^{2}-\left(j-\alpha \frac{\partial}{\partial \alpha}\right)^{2}\right]\left\langle j j\left|\mathrm{e}^{\alpha^{*} \hat{J}_{+}} \rho \mathrm{e}^{\alpha \hat{J}_{-}}\right| j j\right\rangle \\
& =\left(1+\alpha \alpha^{*}\right)^{-2 j}\left[\left(\alpha^{*} \frac{\partial}{\partial \alpha^{*}}\right)^{2}-\left(\alpha \frac{\partial}{\partial \alpha}\right)^{2}+2 j \alpha \frac{\partial}{\partial \alpha}-2 j \alpha^{*} \frac{\partial}{\partial \alpha^{*}}\right]\left\langle j j\left|\mathrm{e}^{\alpha^{*} \hat{J}_{+}} \rho \mathrm{e}^{\alpha \hat{J}_{-}}\right| j j\right\rangle \\
& =\left(1+\alpha \alpha^{*}\right)^{-2 j}\left(\alpha^{*} \frac{\partial}{\partial \alpha^{*}}+\alpha \frac{\partial}{\partial \alpha}-2 j\right)\left(\alpha^{*} \frac{\partial}{\partial \alpha^{*}}-\alpha \frac{\partial}{\partial \alpha}\right)\left\langle j j\left|\mathrm{e}^{\alpha^{*} \hat{J}_{+}} \rho \mathrm{e}^{\alpha \hat{J}_{-}}\right| j j\right\rangle
\end{aligned}
$$


The commutation of the left differential operator with the prefactor generates a further term, while the right one commutes as in (21),

$$
\mathrm{i} \frac{2 j+1}{\tau} \mathcal{G}_{T_{z}} Q_{\rho}=\left(2 j \frac{2 \alpha \alpha^{*}}{1+\alpha \alpha^{*}}-2 j+\alpha \frac{\partial}{\partial \alpha}+\alpha^{*} \frac{\partial}{\partial \alpha^{*}}\right)\left(\alpha^{*} \frac{\partial}{\partial \alpha^{*}}-\alpha \frac{\partial}{\partial \alpha}\right) Q_{\rho} .
$$

Again we replace the complex coordinates by the spherical coordinates,

$$
\begin{aligned}
\alpha \frac{\partial}{\partial \alpha}+\alpha^{*} \frac{\partial}{\partial \alpha^{*}} & =\sin \theta \frac{\partial}{\partial \theta}, \\
1-\frac{2 \alpha \alpha^{*}}{1+\alpha \alpha^{*}}=\frac{1-\alpha \alpha^{*}}{1+\alpha \alpha^{*}} & =\cos \theta,
\end{aligned}
$$

and the generator finally becomes

$$
\mathcal{G}_{T_{z}}=-\tau\left(\cos \theta-\frac{1}{2 j+1} \frac{\partial}{\partial \theta} \sin \theta\right) \frac{\partial}{\partial \varphi} .
$$

\section{B. The torsion generator matrix}

We here calculate the action of the classical and quantum torsion generators on spherical harmonics. Spherical harmonics are separable into $\varphi$ - and $\theta$-dependent parts as

$$
Y_{l m}(\theta, \varphi)=\sqrt{\frac{2 l+1}{4 \pi} \frac{(l-m) !}{(l+m) !}} P_{l}^{m}(\cos \theta) \mathrm{e}^{\mathrm{i} m \varphi}
$$

where $P_{l}^{m}(\cos \theta)$ are the associated Legendre polynomials [11]. At first we focus on the more complicated action of the generators $\mathcal{L}_{T_{z}}$ and $\mathcal{G}_{T_{z}}$ on the $P_{l}^{m}(\cos \theta)$, i.e. for the classical propagator

$$
\cos \theta P_{l}^{m}(\cos \theta)=z P_{l}^{m}(z),
$$

and for the quantum correction (cf. (29))

$$
-\frac{1}{2 j+1} \frac{\partial}{\partial \theta} \sin \theta P_{l}^{m}(\cos \theta)=-\frac{1}{2 j+1}\left(z-\left(1-z^{2}\right) \frac{\mathrm{d}}{\mathrm{d} z}\right) P_{l}^{m}(z) .
$$

By using the following recursion formulae for the $P_{l}^{m}(z)$ [1],

$$
\begin{array}{r}
\left(1-z^{2}\right) \frac{\mathrm{d}}{\mathrm{d} z} P_{l}^{m}(z)=-l z P_{l}^{m}(z)+(l+m) P_{l-1}^{m}(z), \\
(2 l+1) z P_{l}^{m}(z)=(l-m+1) P_{l+1}^{m}+(l+m) P_{l-1}^{m}(z),
\end{array}
$$

one arrives at

$$
z P_{l}^{m}(z)=\frac{l-m+1}{2 l+1} P_{l+1}^{m}(z)+\frac{l+m}{2 l+1} P_{l-1}^{m}(z)
$$

for the classical generator and

$$
-\frac{1}{2 j+1}\left(z-\left(1-z^{2}\right) \frac{\mathrm{d}}{\mathrm{d} z}\right) P_{l}^{m}(z)=-\frac{1}{(2 j+1)(2 l+1)}\left((l+1)(l-m+1) P_{l+1}^{m}(z)-l(l+m) P_{l-1}^{m}(z)\right)
$$

for the quantum correction term. The associated Legendre polynomials can now be replaced by the spherical harmonics (30). Taking into account $\hat{L}_{z} Y_{l m}=m Y_{l m}$, we finally see the classical torsion generator to act on the spherical harmonics as

$$
\mathrm{i} \frac{\mathcal{L}_{T_{z}}}{\tau m} Y_{l m}=\sqrt{\frac{(l-m+1)(l+m+1)}{(2 l+1)(2 l+3)}} Y_{(l+1) m}+\sqrt{\frac{(l-m)(l+m)}{(2 l+1)(2 l-1)}} Y_{(l-1) m},
$$


and the quantum correction as

$$
\mathrm{i} \frac{\mathcal{G}_{T_{z}}-\mathcal{L}_{T_{z}}}{\tau m} Y_{l m}=-\frac{l+1}{2 j+1} \sqrt{\frac{(l-m+1)(l+m+1)}{(2 l+3)(2 l+1)}} Y_{(l+1) m}+\frac{l}{2 j+1} \sqrt{\frac{(l-m)(l+m)}{(2 l-1)(2 l+1)}} Y_{(l-1) m} .
$$

The expression for $\mathcal{G}_{T_{z}} Y_{l m}$ is already given in (14). The matrix elements of $\mathcal{L}_{T_{z}}$ and $\mathcal{G}_{T_{z}}$ in the basis of spherical harmonics can easily be read off from (36) and (14), respectively. It is noteworthy that relative to the classical matrix element the quantum correction, to be read off from (38), is of order $l /(2 j+1)$. Therefore matrix elements of the quantum generator with $l \sim 2 j$ differ significantly from their classical counterparts; most importantly, the quantum correction manifests the finiteness of the expansion of $Q$ at $l=2 j$, in accordance with the remarks after (14).

\section{Husimi torsion propagator}

In order to obtain the propagator matrix (15) the torsion propagator $\exp \left(\mathcal{G}_{T_{z}}\right)$ is to be written in the basis of spherical harmonics. Therefore we start from the eigenrepresentation of the propagator and apply the respective linear transformation. The basis of right-hand eigenfunctions consists of the $(2 j+1)^{2}$ "Husimi functions"

$$
Q_{\left|j m_{1}\right\rangle\left\langle j m_{2}\right|}=\left\langle j \theta \varphi \mid j m_{1}\right\rangle\left\langle j m_{2} \mid j \theta \varphi\right\rangle,
$$

with eigenvalues $\exp \left(-\mathrm{i} \tau\left(m_{1}^{2}-m_{2}^{2}\right) /(2 j+1)\right)$.

The left-hand eigenfunctions lie in the function space dual to the space of Husimi functions. Functions from this

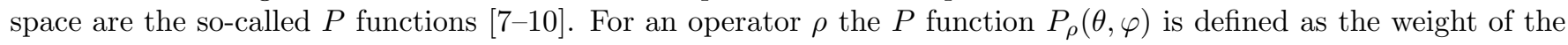
diagonal mixture with respect to coherent states,

$$
\rho=\frac{2 j+1}{4 \pi} \int_{0}^{\pi} \mathrm{d} \theta \sin \theta \int_{0}^{2 \pi} \mathrm{d} \varphi P_{\rho}(\theta, \varphi)|j \theta \varphi\rangle\langle j \theta \varphi| .
$$

In particular, the functions $P_{\left|j m_{1}\right\rangle\left\langle j m_{2}\right|}$ are the left-hand eigenfunctions of $\exp \left(\mathcal{G}_{T_{z}}\right)$ and form a basis of this dual space that is biorthonormal to the basis $Q_{\left|j m_{1}\right\rangle\left\langle j m_{2}\right|}$. Therefore, the eigenrepresentation of the torsion propagator is given by $[10$ ]

$$
\left.\exp \left(\mathcal{G}_{T_{z}}\right)=\sum_{m_{1}, m_{2}=-j}^{j}\left|Q_{\left|j m_{1}\right\rangle\left\langle j m_{2}\right|}\right\rangle\right\rangle \exp \left(-\mathrm{i} \tau \frac{m_{1}^{2}-m_{2}^{2}}{2 j+1}\right)\left\langle\left\langle P_{\left|j m_{1}\right\rangle\left\langle j m_{2}\right|}\right|\right.
$$

wherein the double brackets refer to the scalar product of the classical Hilbert space.

The coefficients $q_{l m}\left(m_{1}, m_{2}\right)$ of the linear transformation connecting Husimi functions and spherical harmonics and the coefficients $p_{l m}\left(m_{1}, m_{2}\right)$ of the inverse transformation can be obtained as 0.0 .10

$$
\begin{aligned}
& q_{l m}\left(m_{1}, m_{2}\right)=\left\langle\left\langle Y_{l m} \mid Q_{\left|j m_{1}\right\rangle\left\langle j m_{2}\right|}\right\rangle\right\rangle=\int \mathrm{d} \theta \mathrm{d} \varphi \sin \theta Y_{l m}^{*} Q_{\left|j m_{1}\right\rangle\left\langle j m_{2}\right|}(\theta, \varphi), \\
& p_{l m}\left(m_{1}, m_{2}\right)=\left\langle\left\langle Y_{l m} \mid P_{\left|j m_{1}\right\rangle\left\langle j m_{2}\right|}\right\rangle\right\rangle \int \mathrm{d} \theta \mathrm{d} \varphi \sin \theta Y_{l m}^{*} P_{\left|j m_{1}\right\rangle\left\langle j m_{2}\right|}(\theta, \varphi) .
\end{aligned}
$$

While the first integral yields the real coefficients (products of Clebsch-Gordan coefficients, incidentally [7,94)

$$
\begin{aligned}
q_{l m}\left(m_{1}, m_{2}\right)= & \delta_{m, m_{1}-m_{2}} \sqrt{4 \pi(2 l+1) \frac{(l-m) !}{(l+m) !}} \sqrt{\left(\begin{array}{c}
2 j \\
j-m_{1}
\end{array}\right)\left(\begin{array}{c}
2 j \\
j-m_{2}
\end{array}\right)} \\
& \times \sum_{k=0}^{l-m} \frac{(-1)^{k+m}(l+m+k) !\left(j+m_{1}\right) !\left(j+k-m_{2}\right) !}{(l-m-k) ! k !(m+k) !(2 j+k+m+1) !}
\end{aligned}
$$

the coefficients $p_{l m}\left(m_{1}, m_{2}\right)$ are proportional to the $q_{l m}\left(m_{1}, m_{2}\right)$,

$$
p_{l m}\left(m_{1}, m_{2}\right)=\frac{\sqrt{4 \pi(2 l+1)}}{(2 j+1) q_{l 0, j j}} q_{l m}\left(m_{1}, m_{2}\right) .
$$


Actually, the above formulae are not particularly useful for numerical purposes as they involve an alternating sum of very large numbers. Easier to employ are recurrence formulae for the coefficients. One formula we used reads

$$
q_{l(m-1)}\left(m_{1}, m_{2}\right)=\frac{1}{w(l,-m)}\left(w\left(j, m_{1}\right) q_{l m}\left(m_{1}+1, m_{2}\right)-w\left(j,-m_{2}\right) q_{l m}\left(m_{1}, m_{2}-1\right)\right),
$$

wherein $w(l, m)=\sqrt{l(l+1)-m(m+1)}$. The recursion starts with

$$
q_{l l}\left(m_{1}, m_{2}\right)=\delta_{l, m_{1}-m_{2}} \sqrt{4 \pi(2 l+1)} \frac{(2 j) ! \sqrt{(2 l) !}}{(2 j+l+1) ! l !} \sqrt{\frac{\left(j+m_{1}\right) !\left(j-m_{2}\right) !}{\left(j-m_{1}\right) !\left(j+m_{2}\right) !}} .
$$

Coefficients with negative values of $m$ can be obtained from ones with positive $m$ through the symmetry relation

$$
q_{l(-m)}\left(m_{1}, m_{2}\right)=(-1)^{m_{1}-m_{2}} q_{l m}\left(m_{1}, m_{2}\right) .
$$

Finally, the propagator matrix in the basis of spherical harmonics is obtained as

$$
\left(\exp \left(\mathcal{G}_{T_{z}}\right)\right)_{l m, l^{\prime} m^{\prime}}=\delta_{m, m^{\prime}} \sum_{m_{1}=\max (-j+m,-j)}^{\min (j, j+m)} q_{l m}\left(m_{1}, m_{1}-m\right) p_{l^{\prime} m}\left(m_{1}, m_{1}-m\right) \exp \left(-\mathrm{i} \tau \frac{2 m m_{1}-m^{2}}{2 j+1}\right) .
$$

[1] J. Weber, F. Haake, and P. Šeba, Phys. Rev. Lett. 85, 3620 (2000)

[2] J. Weber, F. Haake, P. A. Braun, C. Manderfeld, and P. Šeba, J. Phys. A 34, 7195 (2001)

[3] A. V. Andreev and B. L. Altshuler, Phys. Rev. Lett. 75, 902 (1995); O. Agam, B. L. Altshuler, and A. V. Andreev, Phys. Rev. Lett. 75, 4389 (1995); A. V. Andreev,O. Agam, B. D. Simons, and B. L. Altshuler, Phys. Rev. Lett. 76, 1 (1996); A. V. Andreev, B. D. Simons, O. Agam, and B. L. Altshuler, Nuclear Physics B, 482, 536 (1996).

[4] M. R. Zirnbauer in: I.V. Lerner, J.P. Keating, and D.E. Khmelnitskii (eds.), Supersymmetry and Trace Formulae: Chaos and Disorder (Kluwer Academic, New York, 1999)

[5] K.Pance, W. Lu, S. Sridhar, Phys. Rev. Lett. 85, 2737 (2000)

[6] F. Haake, Quantum Signatures of Chaos (Springer, Berlin, 2001)

[7] F. T. Arecchi, E. Courtens, R. Gilmore, H. Thomas, Phys. Rev. A 6, 2211 (1972)

[8] R. Glauber and F. Haake, Phys. Rev. A 13, 357 (1976)

[9] A. M. Peremolov, Generalized Coherent States and Their Applications (Springer, New York, 1986)

[10] C. Manderfeld,Coherent State Representation of the $S U(2)$ Group, http://www.theo-phys.uni-essen.de/tp/u/chris/

[11] M. Abramowitz and I. A. Stegun, Handbook of Mathematical Functions (Dover, New York, 1972)

\begin{tabular}{|r|r|r|r|r|}
\hline$l_{\max }=30$ & $l_{\max }=40$ & $l_{\max }=50$ & $l_{\max }=60$ & $l_{\max }=68$ \\
\hline \hline 0.8018 & 0.8116 & 0.8205 & 0.8103 & 0.8074 \\
\hline 0.7513 & 0.7457 & 0.7547 & 0.7470 & 0.7455 \\
\hline-0.0041 & -0.0063 & -0.0123 & -0.0079 & -0.0027 \\
$\pm \mathrm{i} 0.7225$ & $\pm \mathrm{i} 0.7431$ & $\pm \mathrm{i} 0.7515$ & $\pm \mathrm{i} 0.7517$ & $\pm \mathrm{i} 0.7435$ \\
\hline-0.7391 & -0.7432 & -0.7475 & -0.7510 & -0.7427 \\
\hline-0.6639 & -0.6766 & -0.6746 & -0.6869 & -0.6955 \\
\hline 0.6470 & 0.6628 & 0.6777 & 0.6597 & 0.6727 \\
\hline-0.6218 & -0.6443 & -0.6188 & -0.6377 & -0.6336 \\
\hline 0.5462 & 0.5968 & 0.5847 & 0.5889 & 0.5786 \\
\hline-0.5141 & -0.5406 & -0.5363 & -0.5481 & -0.5448 \\
\hline
\end{tabular}

TABLE I. Frozen nonunimodular eigenvalues of $\mathcal{P}^{(N)}$ with $\tau=10, \beta_{z}=\beta_{y}=1$ at the resolutions $l_{\text {max }}=30,40,50,60,68$. 


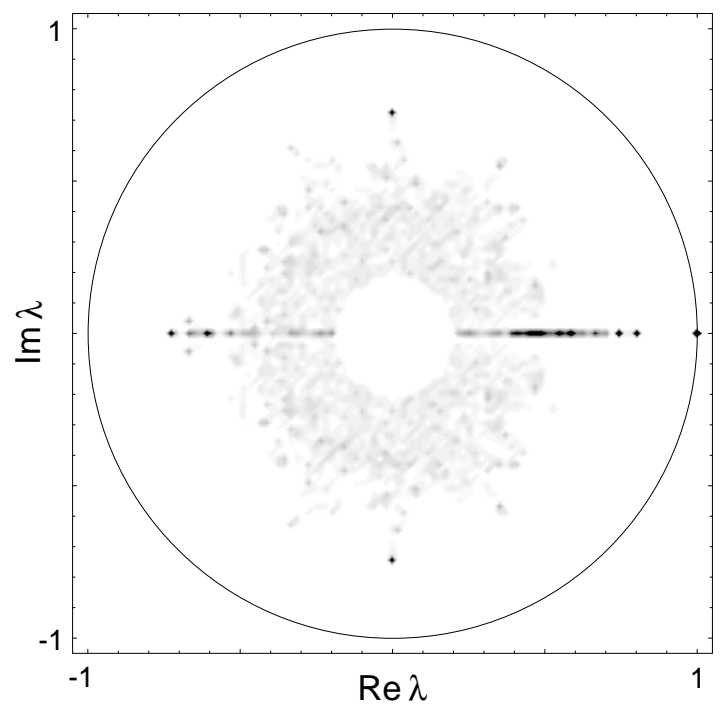

FIG. 1. For the top with $\tau=10, \beta_{z}=\beta_{y}=1$ a histogram from the eigenvalues of $\mathcal{P}^{(N)}$ at resolutions $l_{\text {max }}=20,21 \ldots 70$ in the complex plane has large amplitudes (black) at positions of frozen eigenvalues. Since the mean density of eigenvalues increases drastically near the origin, the disk with radius $1 / 4$ is not included in the histogram.
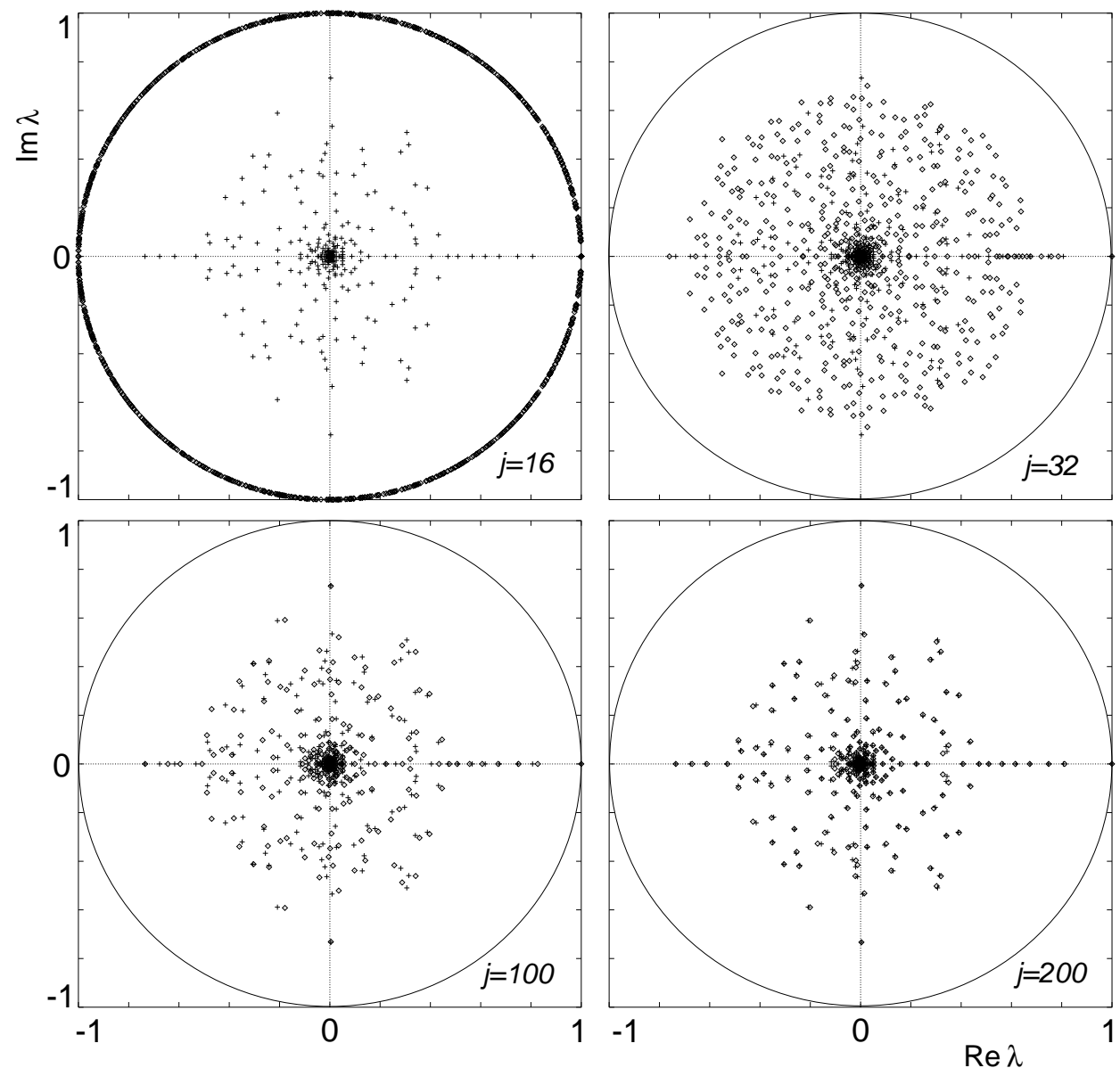

FIG. 2. Eigenvalues of the truncated Husimi $(\diamond)$ and Frobenius-Perron $(+)$ operators $\left(\tau=10, \beta_{z}=1, \beta_{y}=1\right)$ superimposed at $l_{\max }=32$ with the quantum number $j$ taking the values 16 (full resolution), 32, 100, 200. While for $j=16$ the Husimi spectrum is unimodular, for $j=200$ classical and quantum eigenvalues are in good agreement. 


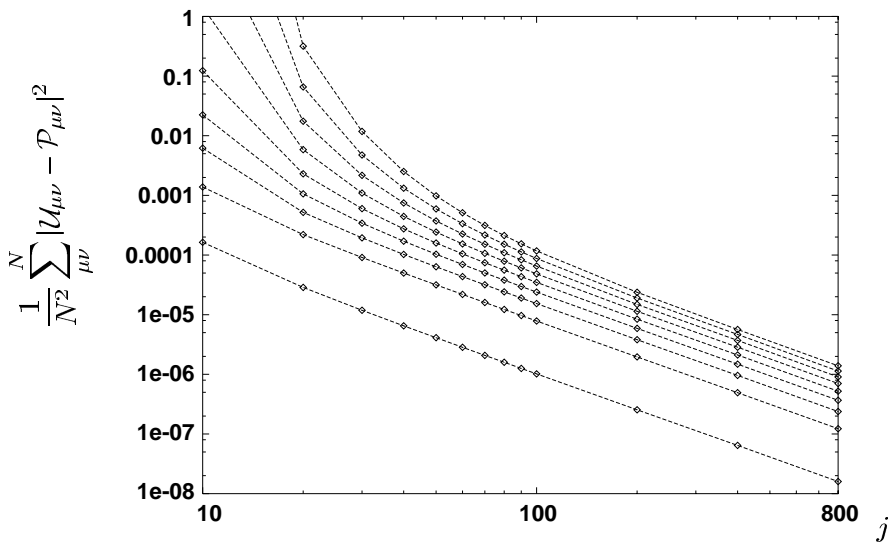

FIG. 3. Mean squared deviation between Husimi and Frobenius-Perron matrix elements of the top $\left(\tau=10, \beta_{z}=1, \beta_{y}=1\right)$ with $l_{\max }=4,6,8,10,12,14,16,18,20$ as a function of $j$ in a double-logarithmic plot. The numerical data suggest a power-law decay of the mean squared deviation as $j \rightarrow \infty$. 